\title{
Determining Reheating Temperature at LHC with Axino or Gravitino Dark Matter
}

\author{
Ki-Young Choi*, Leszek Roszkowski ${ }^{\dagger}$ and Roberto Ruiz de Austri* \\ *Departamento de Física Teórica C-XI and Instituto de Física Teórica UAM/CSIC, \\ Universidad Autónoma de Madrid, Cantoblanco, 28049 Madrid, Spain \\ ${ }^{\dagger}$ Department of Physics and Astronomy, University of Sheffield, \\ Sheffield S3 7RH, England
}

\begin{abstract}
After a period of inflationary expansion, the Universe reheated and reached full thermal equilibrium at the reheating temperature $T_{\mathrm{R}}$. In this talk, based on [1], we point out that, in the context of effective low-energy supersymmetric models, LHC measurements may allow one to determine $T_{\mathrm{R}}$ as a function of the mass of the dark matter particle assumed to be either an axino or a gravitino. An upper bound on their mass and on $T_{\mathrm{R}}$ may also be derived.
\end{abstract}

Keywords: Supersymmetric Effective Theories, Cosmology of Theories beyond the SM, Dark Matter

PACS: $95.35 .+\mathrm{d}, 98.80 . \mathrm{Cq}$

\section{INTRODUCTION}

Recent astrophysical and cosmological observation give precise determination on the relic density of cold dark matter in the range [2] $\Omega_{\mathrm{CDM}} h^{2}=0.104 \pm 0.009$. The well motivated candidate for dark matter (DM) is weakly interacting massive particle (WIMP), especially the lightest neutralino. In addition to the direct and indirect experiments to explore WIMP, the Large Hadron Collider has already started to reveal the secret of TeV energy and is expected to find several superpartners and to determine their properties. In particular, the feasibility was investigated to determine the neutralino's mass $m_{\chi}$ [3, 4] and relic abundance $\Omega_{\chi} h^{2}$ [5, 6] from LHC measurements. An analogous study has also been done in the context of the Linear Collider, where accuracy of a similar determination would be much better [7].

However neutralino is found as an apparently stable state in LHC detectors, but may not be the true LSP and therefore not DM in the Universe. Instead, it could decay into an even lighter and weakly interacting state, the real LSP, outside the detector. Therefore it is necessary to confirm that we need another measurement of neutralino by direct or indirect experiment and also the mass and relic density should be consistent each other.

Moreover, the neutralino relic abundance, as determined at the LHC, may come out convincingly outside the WMAP range. If $\Omega_{\chi} h^{2}$ comes out below WMAP range, several solutions have been suggested which invoke non-standard cosmology, e.g. quintessencedriven kination, while preserving the neutralino as the DM in the Universe. However, if

\footnotetext{
1 Talk given by K.-Y. Choi at International Workshop on Dark Side Of the Universe, 1-5 Jun 2008, Cairo, Egypt.
} 
at the same time direct and indirect DM searches bring null results, or even worse, the lightest super particle turns out to be charged particle such as scalar tau, this will provide a strong indication against the neutralino nature of DM. In fact, these inconsistencies can be perfectly explained with axino or gravitino cold dark matter, which is dubbed as EWIMPs [8].

Therefore this framework give us an opportunity to probe the features of the early Universe, since the relic density of E-WIMPs depends on the reheating temperature $T_{\mathrm{R}}$. Cosmic Lithium problems also can be solved with Gravitino DM [9].

In this talk, based on ref. [1], we investigate the determination of reheating temperature in the E-WIMP scenario using the possible collider measurement of mass and relic density of NLSP, such as neutralino or stau etc.

\section{E-WIMPS AND REHEATING TEMPERATURE $T_{R}$}

The spin-1/2 axino (the fermionic superpartner of an axion) and the spin-3/2 gravitino (the fermionic superpartner of a graviton) are both well-motivated E-WIMPs. The former arises in SUSY extensions of models incorporating the Peccei-Quinn solution to the strong CP problem. The latter is an inherent ingredient of the particle spectrum of supergravity models. The characteristic strength of their interactions with ordinary matter is strongly suppressed by a large mass scale, the Peccei-Quinn scale $f_{a} \sim 10^{11} \mathrm{GeV}$ in the case of axinos and the (reduced) Planck scale $M_{\mathrm{P}} \simeq 2.4 \times 10^{18} \mathrm{GeV}$ for gravitinos. The mass of them are very model dependent and can vary from keV up to $\mathrm{TeV}$ [10] for axino and from $\mathrm{eV}$ to $\mathrm{TeV}$ for gravitino. In this work we want to remain as model-independent as possible and will treat $m_{\tilde{a}}$ and $m_{\widetilde{G}}$ as free parameters. The possibility of axinos as cold DM was pointed out in [11, 12], while axinos as warm DM was considered in [13]. The heavy axino was studied in [14]. The gravitino as a cosmological relic was extensively studied in the literature. For more references refer to [1].

There are two generic ways to produce axinos or gravitinos. One proceeds via scatterings and decay processes of ordinary particles and sparticles in thermal bath. Its efficiency is proportional to their density in the plasma which is a function of $T_{\mathrm{R}}$ (thermal production). The other comes from (out-of-equilibrium) decays of the NLSPs, after their freeze-out, to E-WIMPs (non-thermal production).

The thermal production (TP) of axinos and gravitinos is a function of $T_{\mathrm{R}}$ [12]. For high $T_{\mathrm{R}}$, both are almost proportional to $T_{\mathrm{R}}$. For axino [15]

$$
\Omega_{\tilde{a}}^{\mathrm{TP}} h^{2} \simeq 5.5 g_{s}^{6} \ln \left(\frac{1.108}{g_{s}}\right)\left(\frac{m_{\tilde{a}}}{0.1 \mathrm{GeV}}\right)\left(\frac{10^{11} \mathrm{GeV}}{f_{a}}\right)^{2}\left(\frac{T_{\mathrm{R}}}{10^{4} \mathrm{GeV}}\right),
$$

where $g_{s}$ is temperature-dependent strong coupling constant, which in the above expression is evaluated at $T_{\mathrm{R}}$. Note that, $Y_{\tilde{a}}^{\mathrm{TP}} \propto T_{\mathrm{R}} / f_{a}^{2}$, and for gravitino [16]

$$
\Omega_{\widetilde{G}}^{\mathrm{TP}} h^{2} \simeq 0.27\left(\frac{T_{\mathrm{R}}}{10^{10} \mathrm{GeV}}\right)\left(\frac{100 \mathrm{GeV}}{m_{\widetilde{G}}}\right)\left(\frac{m_{\tilde{g}}(\mu)}{1 \mathrm{TeV}}\right)^{2},
$$

where $m_{\tilde{g}}(\mu)$ stands for the gluino mass evaluated at a scale $\mu \simeq 1 \mathrm{TeV}$. In the axino case, there is a sharp drop-off below $T_{\mathrm{R}} \sim 1 \mathrm{TeV}$ due to Boltzmann suppression factor 

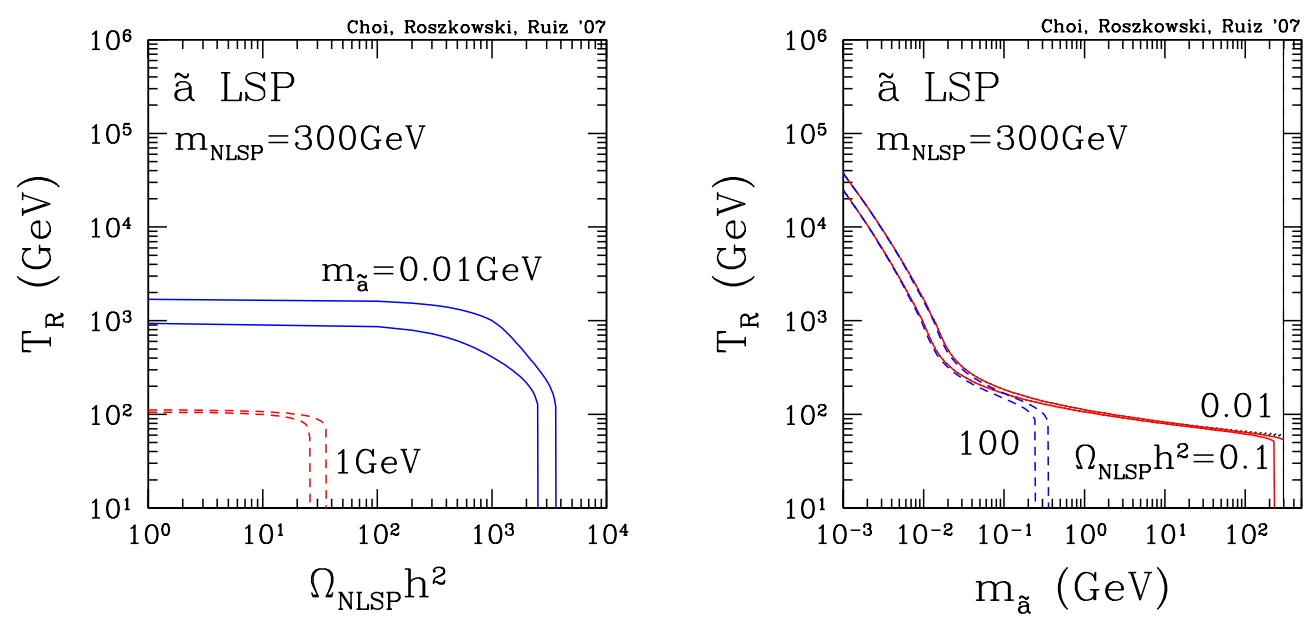

FIGURE 1. Left panel: $T_{\mathrm{R}}$ vs. $\Omega_{\mathrm{NLSP}} h^{2}$ for $m_{\mathrm{NLSP}}=300 \mathrm{GeV}$ and for $m_{\tilde{a}}=0.01 \mathrm{GeV}$ (solid blue) and $m_{\tilde{a}}=1 \mathrm{GeV}$ (dashed red). The bands correspond to the upper and lower limits of dark matter density from WAMP. Right panel: $T_{\mathrm{R}}$ vs. $m_{\tilde{a}}$ for $\Omega_{\mathrm{NLSP}} h^{2}=100$ (dashed blue), 0.1 (solid red) and 0.01 (dotted black). To the right of the solid vertical line the axino is no longer the LSP. In both panels we set $f_{a}=10^{11} \mathrm{GeV}$.

$\exp (-m / T)$, with $m$ denoting here squark and gluino mass; at lower $T_{\mathrm{R}}$ superpartner decay processes become dominant but are less efficient [12].

For the non-thermal production (NTP), the relic abundance is simply given by

$$
\Omega_{\mathrm{LSP}}^{\mathrm{NTP}} h^{2}=\frac{m_{\mathrm{LSP}}}{m_{\mathrm{NLSP}}} \Omega_{\mathrm{NLSP}} h^{2} .
$$

The total abundance of the LSPs is the sum of both thermal and non-thermal production contributions and it is natural to expect that the LSP makes up most of CDM in the Universe, thus we can write

$$
\Omega_{\mathrm{LSP}}^{\mathrm{TP}} h^{2}\left(T_{\mathrm{R}}, m_{\mathrm{LSP}}, m_{\tilde{g}}, m_{\mathrm{NLSP}}, \ldots\right)+\frac{m_{\mathrm{LSP}}}{m_{\mathrm{NLSP}}} \Omega_{\mathrm{NLSP}} h^{2}=\Omega_{\mathrm{LSP}} h^{2}=\Omega_{\mathrm{CDM}} h^{2} \simeq 0.1 .
$$

Once the neutralino NLSP is discovered and its mass is determined at the LHC with some precision, and so also $\Omega_{\mathrm{NLSP}} h^{2}=\Omega_{\chi} h^{2}$, then eq. (4) will provide a relation between $T_{\mathrm{R}}$ and $m_{\mathrm{LSP}}$.

\section{AXINO DARK MATTER}

First we consider axino as the LSP dark matter. Using (4), we can find the relations between the parameters, $T_{\mathrm{R}}, m_{L S P}, m_{N L S P}$, and $\Omega_{\mathrm{NLSP}} h^{2}$. For fixed two parameters, we plot the contour on the space of the other two parameters in figures 1 and 2 .

From figure 1 (right panel), where $m_{\tilde{a}}$ is small, TP dominates, $\Omega_{\tilde{a}}^{\mathrm{TP}} h^{2} \simeq \Omega_{\mathrm{CDM}} h^{2}$, hence we find $T_{\mathrm{R}} \propto f_{a}^{2} / m_{\tilde{a}}$. This relation allows one to derive an upper bound on $T_{\mathrm{R}}$ if we use the fact that axinos have to be heavy enough in order to constitute CDM. Assuming conservatively that $m_{\tilde{a}} \gtrsim 100 \mathrm{keV}$ [12], we find $T_{\mathrm{R}}^{\max }<4.9 \times 10^{5} \mathrm{GeV}$. 

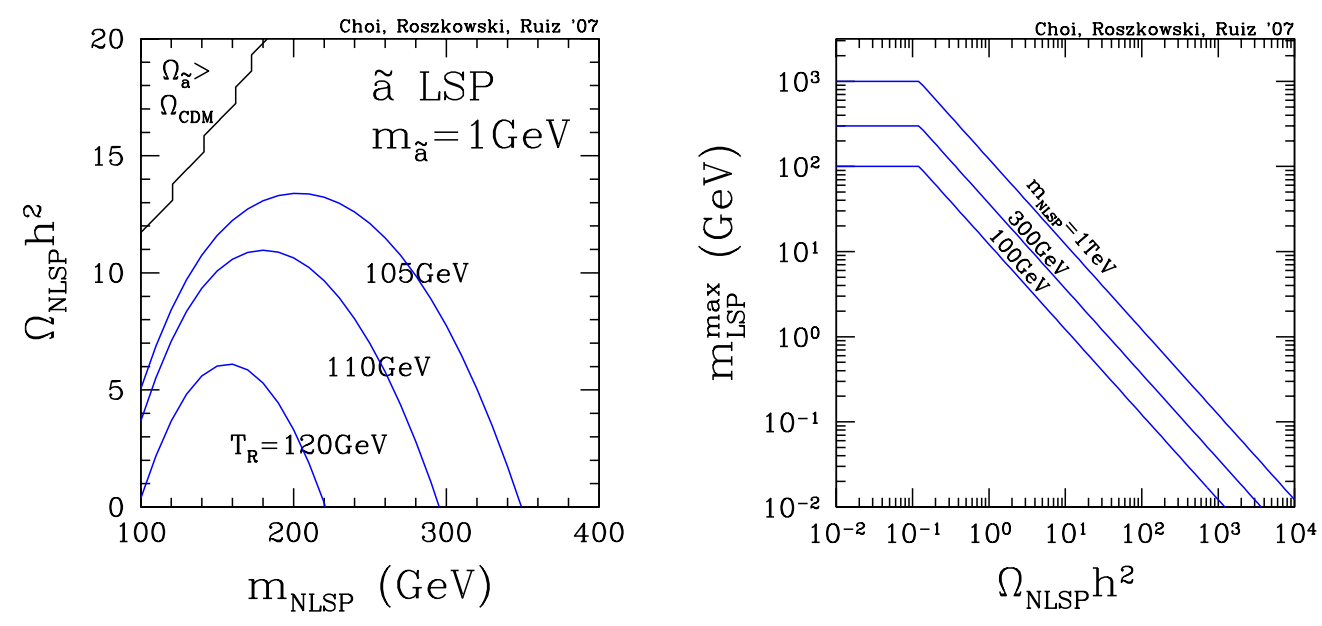

FIGURE 2. Left: Contours of the reheating temperature in the plane of $m_{\mathrm{NLSP}}$ and $\Omega_{\mathrm{NLSP}} h^{2}$ such that $\Omega_{\tilde{a}} h^{2}=\Omega_{\mathrm{CDM}} h^{2}=0.104$. The axino mass is assumed to be $1 \mathrm{GeV}$. Right: Maximum values of $m_{\mathrm{LSP}}$ as a function of $\Omega_{\mathrm{NLSP}} h^{2}$ for representative values of $m_{\mathrm{NLSP}}$. Once both $\Omega_{\mathrm{NLSP}} h^{2}$ and $m_{\mathrm{NLSP}}$ are determined from experiment, the upper bound on $m_{\mathrm{LSP}}$ can be derived. The plot applies both to the axino and to the gravitino LSP.

At larger $m_{\tilde{a}}$ the NTP contribution becomes dominant and the dependence on $T_{\mathrm{R}}$ is lost, but in this regime the LSP mass becomes largest, this allows one to derive an upper bound on $m_{\tilde{a}}$. This is shown in fig. 2 (right panel). Note that fig. 2)(right panel) is actually applied to both the axino and the gravitino LSP since it follows from eq. (3).

\section{GRAVITINO DARK MATTER}

For gravitino LSP, the analogous plots are shown in figures 3 and 4 . For gravitino LSP, Big Bang Nucleosynthesis (BBN) gives strong constraint since the lifetime of NLSP is around $1 \mathrm{sec}$ to $10^{12} \mathrm{sec}$ due to the suppressed interaction. The neutralino NLSP is almost excluded with $m_{\widetilde{G}} \gtrsim 1 \mathrm{GeV}$ due to BBN [17, 18].

While $Y_{\widetilde{a}}^{\mathrm{TP}}$ is independent of the axino mass, in the gravitino case $Y_{\widetilde{G}}^{\mathrm{TP}} \propto 1 / m_{\widetilde{G}}^{2}$. Thus $\Omega_{\tilde{a}}^{\mathrm{TP}} h^{2} \propto m_{\tilde{a}} T_{\mathrm{R}}$ while $\Omega_{\widetilde{G}}^{\mathrm{TP}} h^{2} \propto T_{\mathrm{R}} / m_{\widetilde{G}}$. In other words, if TP dominates, $\Omega_{\widetilde{G}}^{\mathrm{TP}} h^{2} \simeq 0.1$, we find $T_{\mathrm{R}} \propto m_{\widetilde{G}}$. And then NTP dominates, $T_{\mathrm{R}}$ drops down as shown in figure 3 (right panel). The turnover between the TP and NTP dominance allows one to derive a conservative upper bound $T_{\mathrm{R}}^{\max }$ which, unlike for the axino CDM, even without knowing the gravitino mass. This is plotted in fig. (4)(right panel).

For stau NLSP case, in our numerical example in the right panel of fig. 3, with $m_{\tilde{\tau}}=300 \mathrm{GeV}$, where we have also taken $m_{\chi}=477 \mathrm{GeV}$, the condition $\tau_{\tilde{\tau}}>10^{3} \mathrm{sec}$ implies $m_{\widetilde{G}} \lesssim 2 \mathrm{GeV}$ and $T_{\mathrm{R}} \lesssim 9 \times 10^{6} \mathrm{GeV}$. Increasing $m_{\tilde{\tau}}$ to $1 \mathrm{TeV}$ and $m_{\chi}$ to $1.5 \mathrm{TeV}$ leads to $m_{\widetilde{G}} \lesssim 40 \mathrm{GeV}$ and $T_{\mathrm{R}} \lesssim 4 \times 10^{8} \mathrm{GeV}$. More detailed study on $T_{\mathrm{R}}$ bound with stau NLSP considering only thermal production and using the constraint from bound state effects can be seen in [19]. 

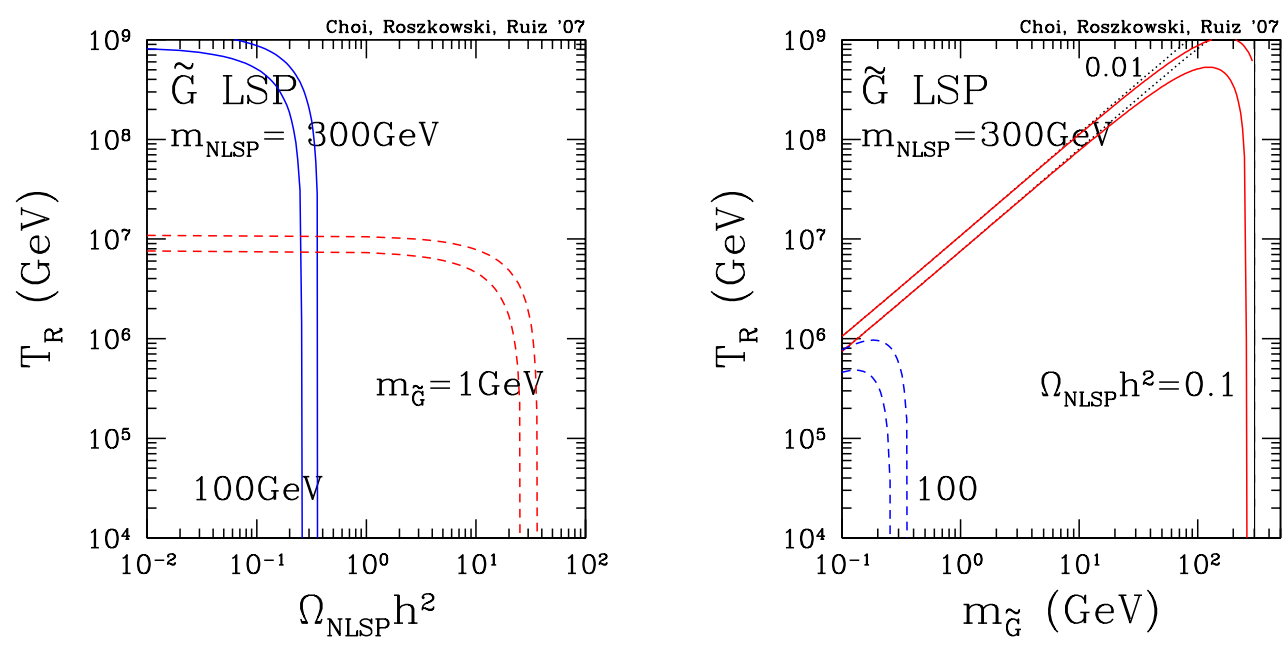

FIGURE 3. Left panel: $T_{\mathrm{R}}$ vs. $\Omega_{\mathrm{NLSP}} h^{2}$ for $m_{\mathrm{NLSP}}=300 \mathrm{GeV}$ and for $m_{\widetilde{G}}=0.01 \mathrm{GeV}$ (solid blue) and $m_{\widetilde{G}}=1 \mathrm{GeV}$ (dashed red). The bands correspond to the upper and lower limits of dark matter density from WMAP. Right panel: $T_{\mathrm{R}}$ vs. $m_{\widetilde{G}}$ for $\Omega_{\mathrm{NLSP}} h^{2}=100$ (dashed blue), 0.1 (solid red) and 0.01 (dotted black). To the right of the solid vertical line the gravitino is no longer the LSP.
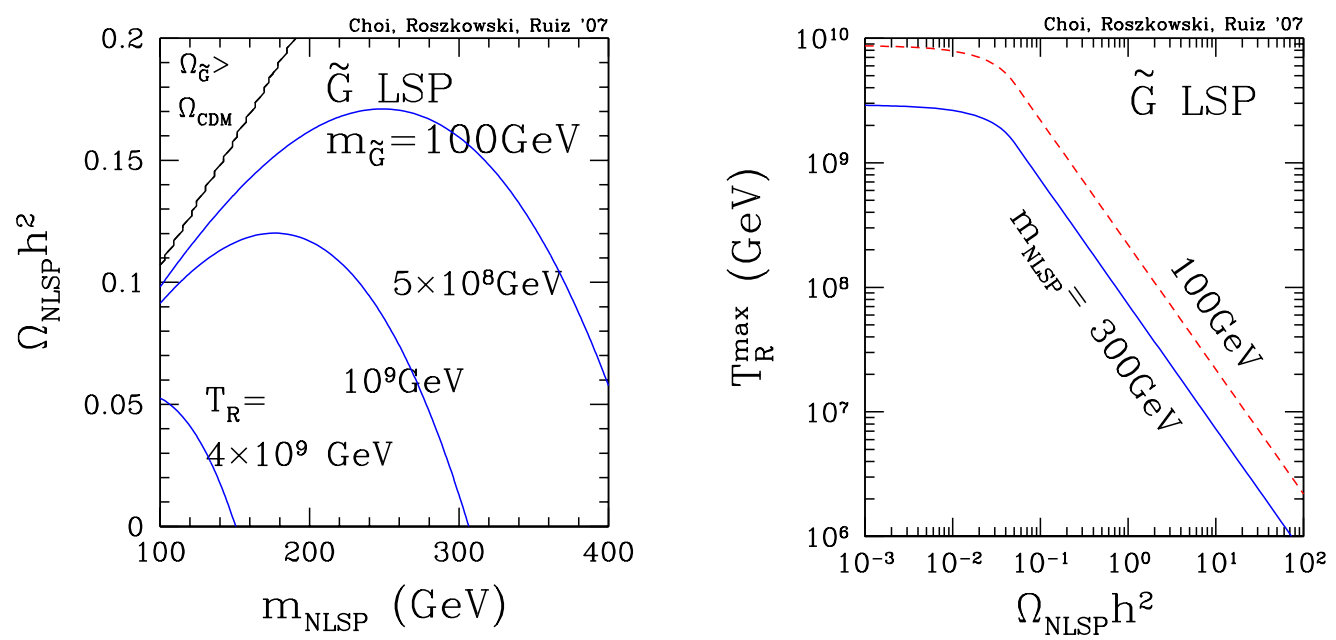

FIGURE 4. Left: Contours of the reheating temperature in the plane of $m_{\mathrm{NLSP}}$ and $\Omega_{\mathrm{NLSP}} h^{2}$ such that $\Omega_{\widetilde{G}} h^{2}=\Omega_{\mathrm{CDM}} h^{2}=0.104$. The gravitino mass is assumed to be $100 \mathrm{GeV}$. Right: Maximum reheating temperature $T_{\mathrm{R}}^{\max }$ vs. NLSP relic density $\Omega_{\mathrm{NLSP}} h^{2}$ with gravitino DM for NLSP mass $m_{\mathrm{NLSP}}=100 \mathrm{GeV}$ (dashed red) and $300 \mathrm{GeV}$ (solid blue).

\section{SUMMARY}

We studied the possible determination of reheating temperature with axino or gravitino LSP dark matter. We find that once we know the mass of NLSP and other parameters determining its relic abundance from collider data we can give the reheating temperature depending on the mass of LSP. Even though the relic abundance of the NLSP is not 
measured precisely, if the order of magnitude is much smaller or larger than WMAP range then we can obtain conservative bound on the reheating temperature.

Note Added: Recently, and long after our work was published, a paper appeared [19] which, using a different set of variables (NLSP stau lifetime and mass, instead of our gravitino mass and NLSP mass) and neglecting non-thermal contribution to $\Omega h^{2}$, rederived several of our results. Specifically, with stau mass around $1 \mathrm{TeV}$, an upper limit on the reheating temperature of $T_{\mathrm{R}} \lesssim 10^{8} \mathrm{GeV}$ was obtained, in agreement with ours.

\section{ACKNOWLEDGMENTS}

K.-Y.C. is supported by the Ministerio de Educacion y Ciencia of Spain under Proyecto Nacional FPA2006-05423 and by the Comunidad de Madrid under Proyecto HEPHACOS, Ayudas de I+D S-0505/ESP-0346. L.R is partially supported by the EC 6th Framework Programmes MRTN-CT-2004-503369 and MRTN-CT-2006-035505. R.RdA is supported by the program "Juan de la Cierva" of the Ministerio de Educación y Ciencia of Spain.

\section{REFERENCES}

1. K. Y. Choi, L. Roszkowski and R. Ruiz de Austri, JHEP 0804 (2008) 016 [arXiv:0710.3349].

2. D. N. Spergel, et al. [WMAP Collaboration], Astrophys. J. Supp. 170, 377 (2007) [astro-ph/0603449].

3. W. S. Cho, K. Choi, Y. G. Kim and C. B. Park, Phys. Rev. Lett. 100 (2008) 171801 [arXiv:0709.0288]]; W. S. Cho, K. Choi, Y. G. Kim and C. B. Park, JHEP 0802 (2008) 035.

4. A. J. Barr, G. G. Ross and M. Serna, arXiv:0806.3224 [hep-ph].

5. G. Polesello and D. R. Tovey, JHEP 0405, 071 (2004) [hep-ph/0403047]. See also B. C. Allanach, G. Belanger, F. Boudjema, A. Pukhov, JHEP 0412, 020 (2004) [hep-ph/0410091v2] and E. A. Baltz, M. Battaglia, M. E. Peskin, T. Wizansky, Phys. Rev. D 74, 103521 (2006) [hep-ph/0602187v4].

6. M. M. Nojiri, G. Polesello and D. R. Tovey, JHEP 0603, 063 (2006) [hep-ph/0512204].

7. M. Battaglia, hep-ph/0410123v1; A. Birkedal et al., hep-ph/0507214, T. Moroi, Y. Shimizu, A. Yotsuyanagi, Phys.Lett. B625 (2005) 79-87 [hep-ph/0505252v2].

8. K. Y. Choi and L. Roszkowski, AIP Conf. Proc. 805 (2006) 30 [arXiv:hep-ph/0511003].

9. K. Jedamzik, K. Y. Choi, L. Roszkowski and R. Ruiz de Austri, JCAP 0607 (2006) 007 [arXiv:hep-ph/0512044].

10. E.J. Chun, J.E. Kim and H.P. Nilles, Phys. Lett. B 287, 123 (1992); E.J. Chun and A. Lukas, Phys. Lett. B 357, 43 (1995).

11. L. Covi, J. E. Kim and L. Roszkowski, Phys. Rev. Lett. 82, 4180 (1999) [hep-ph/9905212].

12. L. Covi, H. B. Kim, J. E. Kim and L. Roszkowski, JHEP 0105, 033 (2001) |hep-ph/0101009|.

13. K. Rajagopal, M.S. Turner and F. Wilczek, Nucl. Phys. B 358, 447 (1991).

14. K. Y. Choi, J. E. Kim, H. M. Lee and O. Seto, Phys. Rev. D 77 (2008) 123501.

15. A. Brandenburg and F. D. Steffen, JCAP 0408, 008 (2004) [hep-ph/0405158].

16. M. Bolz, A. Brandenburg and W. Buchmüller, Nucl. Phys. B 606, $518(2001)$ [hep-ph/0012052].

17. J.L. Feng, A. Rajaraman and F. Takayama, Phys. Rev. Lett. 91, 011302 (2003) [hep-ph/0302215]; J.L. Feng, A. Rajaraman and F. Takayama, Phys. Rev. D 68, 063504 (2003) [hep-ph/0306024]; J. L. Feng, S. f. Su and F. Takayama, Phys. Rev. D 70, 063514 (2004) [hep-ph/0404198] and Phys. Rev. D 70, 075019 (2004) [hep-ph/0404231].

18. L. Roszkowski, R. Ruiz de Austri and K. Y. Choi, JHEP 0508 (2005) 080 [arXiv:hep-ph/0408227]; D. G. Cerdeno, K. Y. Choi, K. Jedamzik, L. Roszkowski and R. Ruiz de Austri, JCAP 0606 (2006) 005 [arXiv:hep-ph/0509275].

19. F. D. Steffen, Phys. Lett. B 669 (2008) 74 [arXiv:0806.3266 [hep-ph]]. 